\title{
Post-traumatic hydrocephalus following decompressive craniectomy: how well can it be predicted?
}

\author{
Varidh Katiyar $^{1} \cdot$ Sundarakrishnan Dharanipathy ${ }^{1} \cdot$ Hitesh Gurjar $^{1} \cdot$ Zainab Vora $^{1} \cdot$ Ravi Sharma ${ }^{1}$
}

Received: 15 August 2018 / Accepted: 20 September 2018 / Published online: 2 October 2018

(C) Springer-Verlag GmbH Austria, part of Springer Nature 2018

Dear Editor,

We studied with keen interest the article by Nasi et al. regarding the risk factors for post-traumatic hydrocephalus (PTH) after decompressive craniectomy in traumatic brain injury patients and their 6-month clinical outcomes [2].

We commend the authors on undertaking an evaluation of these risk factors as early prediction of hydrocephalus may facilitate early anticipatory management and result in a better long-term outcome vis-à-vis a late responsive intervention. In this study, the authors have found radiological finding of interhemispheric hygroma and delayed cranioplasty to be significantly associated with development of post-traumatic hydrocephalus.

The predictive potential of interhemispheric hygroma has been reported by other authors as well. According to these findings, subdural hygroma preceded the development of PTH and can be a good predictor for the same. One of the studies by Kaen et al. [1] reported a sensitivity of $94 \%$ and specificity of $96 \%$. However, Kaen et al. [1] also observed the appearance of PTH after the resolution of subdural hygroma. The authors failed to acknowledge this interesting finding and have not commented on the status of subdural hygroma in their surgical series at the time of development of PTH.

There have been several studies evaluating different aspects of debate between early and late cranioplasty. It is traditionally classified at an arbitrary cutoff of 3 months. The authors have used this cutoff of 3 months only which seems to be counterintuitive for the purpose of this study because the as-

This article is part of the Topical Collection on Brain Trauma

Hitesh Gurjar

hiteshgurjar@gmail.com

1 Department of Neurosurgery, All India Institute of Medical Sciences, New Delhi, India sessment of post-traumatic hydrocephalus was done at 1 month after decompressive craniectomy in this study. Thus, it must have been classified at 1 month to understand the impact cranioplasty has on the development of PTH. An early cranioplasty done between 1 and 3 months after decompressive craniectomy is not expected to benefit a patient in terms of development of PTH at 1 month vis-à-vis delayed cranioplasty.

In this study, there were 37 patients who developed PTH out of whom 3 underwent early cranioplasty. The authors have described 3 patients who developed PTH and were managed by cranioplasty and lumbar drain insertion. It must have been made clear that these 3 patients are the same or not. If they are, then cranioplasty is more of a therapeutic intervention done for PTH rather than a risk factor for the same. It does not preclude the predictive potential of early cranioplasty but to assess that either the association must be evaluated with PTH at $>3$ months or the cutoff time for cranioplasty must be reduced accordingly. The choice between ventriculoperitoneal shunt and urgent cranioplasty and lumbar drain insertion as the therapeutic modality should also have been explained.

\section{Compliance with ethical standards}

Conflict of interest The authors declare that they have no conflict of interest.

\section{References}

1. Kaen A, Jimenez-Roldan L, Alday R, Gomez PA, Lagares A, Alén JF, Lobato RD (2010) Interhemispheric hygroma after decompressive craniectomy: does it predict posttraumatic hydrocephalus? J Neurosurg 113(6):1287-1293

2. Nasi D, Gladi M, Di Rienzo A, di Somma L, Moriconi E, Iacoangeli M, Dobran M (2018) Risk factors for post-traumatic hydrocephalus following decompressive craniectomy. Acta Neurochir. https://doi. org/10.1007/s00701-018-3639-0 